\title{
Inner-shell single and double ionization potentials of aminophenol isomers
}

\author{
Nikolai V. Kryzhevoi, ${ }^{1, a)}$ Robin Santra, ${ }^{2,3}$ and Lorenz S. Cederbaum ${ }^{1}$ \\ ${ }^{1}$ Theoretical Chemistry, Institute of Physical Chemistry, Heidelberg University, 69120 Heidelberg, Germany \\ ${ }^{2}$ Center for Free-Electron Laser Science, DESY, 22607 Hamburg, Germany \\ ${ }^{3}$ Department of Physics, University of Hamburg, 20355 Hamburg, Germany
}

(Received 9 May 2011; accepted 20 July 2011; published online 22 August 2011)

\begin{abstract}
A comprehensive study of single and double core ionization potentials of the aminophenol molecule is reported. The role of relaxation, correlation, relativistic, and basis set effects in these potentials is clarified. Special attention is paid to the isomer dependence of the single and double core ionization potentials. Some of them are also compared with the respective values of the phenol and aniline molecules. It is shown that the core level single ionization potentials of the para-, meta-, and ortho-aminophenol molecules differ only slightly from each other, rendering these structural isomers challenging to distinguish for conventional x-ray photoelectron spectroscopy. In contrast, the energy needed to remove two core electrons from different atoms depends noticeably on the mutual arrangement and even on the relative orientations of the hydroxyl and amine groups. Together with the electrostatic repulsion between the two core holes, relaxation effects accompanying double core ionization play a crucial role here. The pronounced sensitivity of the double ionization potentials, therefore, enables a spectroscopic characterization of the electronic structure of aminophenol isomers by means of x-ray two-photon photoelectron spectroscopy. () 2011 American Institute of Physics. [doi:10.1063/1.3624393]
\end{abstract}

\section{INTRODUCTION}

X-ray two-photon photoelectron spectroscopy (XTPPS) (Ref. 1) is a promising tool for probing the electronic structure of molecules. By measuring the kinetic energies of two photoelectrons generated via sequential absorption of two photons from a femtosecond $\mathrm{x}$-ray pulse, core level double ionization potentials (DIPs) of a chemical compound can be obtained. The duration of the pulse plays an important role and must be short enough to exclude Auger decay of the first core vacancy before the second x-ray photon is absorbed. The utility of XTPPS was first demonstrated theoretically by the example of the para-aminophenol (p-AP) molecule ${ }^{1}$ whose XTPPS spectrum was constructed by making use of pulse parameters of the Linac Coherent Light Source (LCLS). ${ }^{2,3}$ The first experimental production of double core hole states with the LCLS was performed recently for $\mathrm{Ne}$ (Ref. 4) and $\mathrm{N}_{2} .5,6$

Molecular core level doubly ionized states with both vacancies on the same atomic site can be formed without intense x-ray pulses, e.g., indirectly through core-core-core Auger decay. Extensive explorations of these states in the past (see, e.g., Refs. 7-9) have led, in particular, to establishing the Auger parameter or so-called "Wagner plots" concept ${ }^{10}$ (see also the review paper ${ }^{11}$ ) which still represents a convenient tool to study the chemical state of core ionized species including the relaxation effects. ${ }^{12,13}$ Nowadays, molecular doubly ionized states with core vacancies on the same atomic site can also be formed and identified by means of synchrotron radiation combined with multielectron coincidence detection ${ }^{14-16}$ that extends the application of the above concept to the firstrow atoms and to the $\mathrm{K}$-shell vacancies.

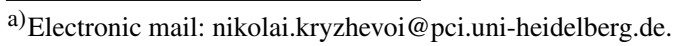

One may, however, encounter a situation when different chemical compounds can hardly be distinguished based on their measured core level single ionization potentials (IPs). Measuring one atomic site DIPs does not improve this situation as the sensitivity of such DIPs to the local environment and to the bonding properties is not critically better than the sensitivity of the single IPs. In contrast, doubly ionized states with core vacancies on different atomic centers and the respective DIPs are noticeably more sensitive to the chemical environment than the single and one-site double IPs as shown by Cederbaum et al. ${ }^{17,18}$ This fact gave rise to a number of subsequent studies aimed at clarifying different aspects of double core ionization and properties of two-site DIPs. ${ }^{19-22}$ The interest in this subject, however, faded away because experimental techniques capable of measuring two-site DIPs were not available at that time.

The development of $\mathrm{x}$-ray free-electron lasers and their application in XTPPS has inspired a renewed interest in double core ionization potentials. Besides the measurements mentioned above, several other XTPPS experiments have recently been conducted and the results obtained are currently being analyzed. For a number of small molecules, $K$-shell DIPs have been calculated and various physical contributions to these potentials were thoroughly analyzed. ${ }^{23,24}$ A method was proposed for extracting relaxation energies from measured XTPPS spectra.

In our first paper on XTPPS, the para-aminophenol molecule was considered. ${ }^{1}$ The $\mathrm{OH}$ and $\mathrm{NH}_{2}$ groups in aminophenol can also be found in meta- and orthocombinations. The meta-aminophenol (m-AP) and orthoaminophenol (o-AP) molecules have, respectively, two isomers which we call $\mathrm{A}$ and $\mathrm{B}$ and which are distinguished by different relative orientations of the hydroxyl and amine 


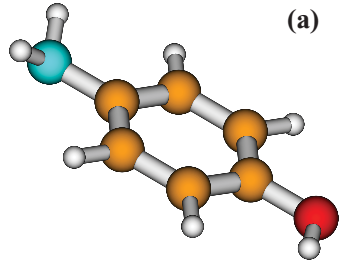

(b)
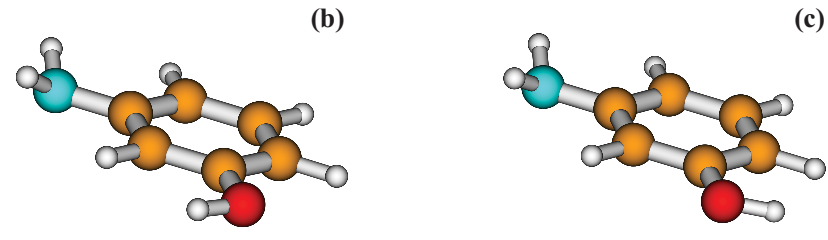

(d)

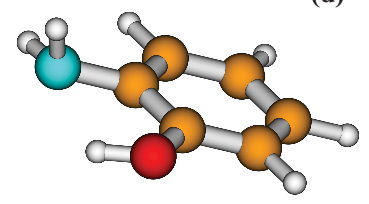

(e)

FIG. 1. Optimized ground-state geometries of five aminophenol isomers: para-aminophenol (a); the A and B isomers of meta-aminophenol ((b) and (c), respectively); the A and B isomers of ortho-aminophenol ((d) and (e), respectively).

groups: $\mathrm{OH}$ points towards $\mathrm{NH}_{2}$ in the isomer $\mathrm{A}$, and away from $\mathrm{NH}_{2}$ in the isomer $\mathrm{B}$. It is expected that differences in electronic and geometric structures of the distinct aminophenol isomers are better reflected in two-site DIPs than in single IPs. The calculations presented here show that this expectation is realized.

\section{COMPUTATIONAL DETAILS}

The electronic structure studies of aminophenol isomers were carried out in the present work at optimized molecular ground state equilibrium geometries. The geometry optimization was performed using the second-order Møller-Plesset perturbation theory in conjunction with the aug-cc-pVDZ basis sets ${ }^{25}$ within the GAUSSIAN03 program suite. ${ }^{26}$ The computed structures are shown in Fig. 1.

Before analyzing the isomer dependence of the innershell single and double ionization potentials of aminophenol, we clarified the role of relaxation, correlation as well as basis set, and relativistic effects in these potentials. The corresponding calculations were done for the representative para-aminophenol (p-AP) molecule.

In the beginning, we obtained an overview of the complete single and double core ionization spectra of p-AP by means of Green's function or propagator methods within algebraic diagrammatic construction (ADC) schemes. ${ }^{27,28}$ To make the ADC and other post-Hartree-Fock calculations feasible, we had to use the relatively small $6-31 G^{*}$ basis sets for oxygen and nitrogen and 6-31G for the other atoms. ${ }^{29}$ The $n$ th-order $\mathrm{ADC}$ scheme, $\operatorname{ADC}(n)$, is complete through $n$th order of the perturbation expansion of the corresponding Green's function and includes in addition infinite summations over certain classes of Feynman diagrams. The ionic main states are thus treated consistently through $n$th order in the $\operatorname{ADC}(n)$ scheme while the secondary excited states are treated consistently through lower orders. In the ADC(4) scheme, which we employed to calculate single core ionization spectra, these secondary states comprise single and double excitations in the presence of a core vacancy. In the ADC(2) scheme used for calculating double core ionization spectra, the secondary states are only single excitations.

Then the selected inner-shell single and double ionization potentials were also calculated by means of the $\triangle \mathrm{SCF}$ (Ref. 30) and singles-and-doubles $\triangle \mathrm{MRCI}$ (multireference configuration interaction) methods where the ionization energies are obtained as the differences between the total energies of the ionic and ground states. The reference sets used in the MRCI calculations include all configurations with the CI coefficients larger than 0.04. Besides the respective ground and main ionic configurations, these are the dominant $\pi \pi^{*}$ double excitations. By virtue of such calculations, we gained access to the relaxation and correlation energies associated with single and double core ionization.

In addition, we estimated relativistic effects by performing relativistic calculations with the zeroth-order regular approximation (ZORA). Like other approximate two-component approaches (e.g., the Douglas-Kroll-Hess method), ZORA exploits a transformed four-component Dirac Hamiltonian. This relativistic method is known to be accurate, numerically stable and cheap and, therefore, we have chosen it to estimate relativistic effects (see, e.g., Refs. 31,32 and references therein).

By utilizing the $\triangle \mathrm{SCF}$ method and the cc-pVXZ $(\mathrm{X}=\mathrm{D}, \mathrm{T}, \mathrm{Q})$ basis sets, ${ }^{25}$ we got insight into the basis set dependence of the inner-shell IPs and DIPs and, moreover, have established the complete-basis-set limits of these potentials at the $\triangle \mathrm{SCF}$ level through an extrapolation of the results obtained in the sequence of computations.

Finally, we studied the isomer dependence of the innershell IPs and DIPs of aminophenol. The respective calculations were carried out for all five aminophenol isomers using the $\triangle \mathrm{SCF}$ method in conjunction with the cc-pVQZ basis set. The core level IPs and DIPs of the phenol and aniline molecules were also calculated for comparison.

The ADC results have been obtained by utilizing the respective ADC $\operatorname{codes}^{33,34}$ interfaced to the GAMESS-UK quantum chemistry package. ${ }^{35}$ Both non-relativistic and relativistic $\triangle \mathrm{SCF}$ and $\triangle \mathrm{MRCI}$ calculations have been performed using GAMESS-UK.

\section{INNER-SHELL SINGLE AND DOUBLE IONIZATION OF THE PARA-AMINOPHENOL MOLECULE}

\section{A. Overview of the single and double core ionization spectra}

Inner-shell single and double ionization potentials of para-aminophenol calculated with the $\operatorname{ADC}(4)$ and $\operatorname{ADC}(2)$ 


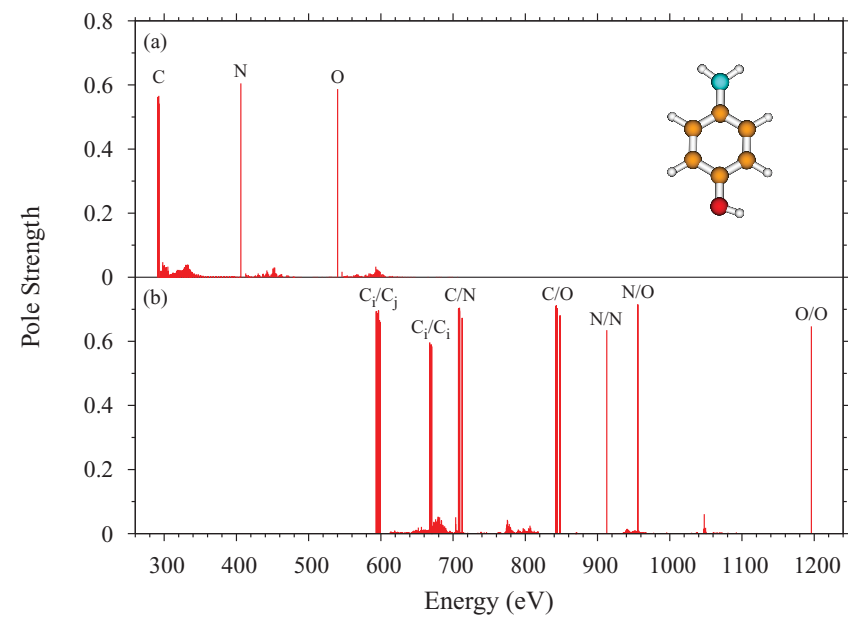

FIG. 2. $K$-shell single (a) and double (b) ionization potentials of paraaminophenol calculated using many-body Green's function methods at the $\mathrm{ADC}(4)$ and $\mathrm{ADC}(2)$ levels, respectively.

methods, respectively, are presented in Fig. 2 as stick spectra. The spectra of other aminophenol isomers look similar and we, therefore, do not show them.

The single ionization spectrum [Fig. 2(a)] exhibits three groups of main lines at about 292, 406, and $540 \mathrm{eV}$ corresponding to $K$-shell ionization of the atomic species $\mathrm{C}, \mathrm{N}$, and $\mathrm{O}$, respectively. Since aminophenol has six non-equivalent $\mathrm{C}$ atoms, there are six distinct $\mathrm{C} 1 s$ main lines in the respective carbon spectral group. However, they can hardly be distinguished in Fig. 2(a) because of rather small energy splittings between them.

The inner-shell double ionization spectrum [Fig. 2(b)] is much richer than the single ionization spectrum. It exhibits seven groups of main lines. For instance, a $1 s$ vacancy on one $\mathrm{C}$ atom may be accompanied by a $1 s$ vacancy on another $\mathrm{C}$ atom. This is symbolized by $\mathrm{C}_{i} / \mathrm{C}_{j}$ in Fig. 2 (b); $\mathrm{C}_{i} / \mathrm{C}_{i}$ indicates that both $1 s$ holes reside on the same $\mathrm{C}$ atom. It is apparent that the chemical shifts for all groups characterized by at least one $\mathrm{C} 1 s$ hole are more pronounced than for the $\mathrm{C} 1 s$ single ionization lines in Fig. 2(a).

The removal of a core electron induces considerable relaxation of valence electrons. The presence of numerous satellites in core level spectra is indicative of this reorganization. In the single core ionization spectrum of p-AP, the pole strengths of the main lines are only $0.55-0.6$ (for comparison, the pole strengths of main lines corresponding to outer-valence ioniza- tion usually exceed 0.8 ), meaning that $40 \%-45 \%$ of the total spectral intensity is distributed between shake-up and shakeoff satellites. These satellites are found in an energy region ranging from a few to several tens of $\mathrm{eV}$ above the respective main lines as seen in Fig. 2(a).

A rich satellite structure is also found in the double core ionization spectrum of p-AP as seen in Fig. 2(b). This is expected since a removal of two core electrons from a system induces even larger relaxation effects than in the case of single core ionization. We note, however, that the pole strengths of the main lines in the calculated double core ionization spectrum of p-AP are counterintuitively slightly higher. The total intensity acquired by satellites is thus lower and the satellites themselves are more separated from the respective main lines than in the single core ionization spectrum. However, we expect the contrary to apply and the recent experiments ${ }^{15}$ support these expectations. This deficiency can be explained by the inability of the ADC(2) method to fully account for the relaxation effects induced by double core ionization. In order to describe these effects correctly and thus to obtain accurate energies and pole strengths of both the main and satellite lines in the double core ionization spectra, one would need, like discussed above for the case of single ionization, a fourth-order ADC scheme for the particle-particle propagator. This method is not yet available.

\section{B. Decomposition of core level IPs and DIPs}

Having gained insight into how the complete single and double core ionization spectra of p-AP look like, we address now the ionization potentials corresponding to the main single and double core hole states and discuss their constituent parts.

Conventionally, the single ionization potential related to a vacancy $S^{-1}$ is decomposed as follows:

$$
\operatorname{IP}\left(S^{-1}\right)=-\varepsilon_{S}-R\left(S^{-1}\right)-C\left(S^{-1}\right),
$$

where the orbital energy with negative sign, $-\varepsilon_{S}$, is an approximation for the ionization potential resulted from Koopmans' theorem (the frozen orbital approximation), and the terms $R\left(S^{-1}\right)$ and $C\left(S^{-1}\right)$ describe corrections due to relaxation and correlation effects, respectively.

The ionization potentials of the para-aminophenol molecule predicted by Koopmans' theorem are given in the first row of Table I. By making use of these results and of

TABLE I. Selected $K$-shell single and double IPs of the para-aminophenol molecule, the repulsion, relaxation and correlation energies as well as relativistic corrections calculated as described in the text. The 6-31G* basis set was used for the oxygen and nitrogen atoms. Carbons and hydrogens were described with the 6-31G basis sets. All values are in $\mathrm{eV}$. The carbon $\mathrm{C}_{1}$ and $\mathrm{C}_{4}$ atoms are adjacent to the $\mathrm{OH}$ and $\mathrm{NH}_{2}$ groups, respectively.

\begin{tabular}{|c|c|c|c|c|c|c|c|c|c|c|}
\hline & $\mathrm{O}$ & $\mathrm{N}$ & $\mathrm{C}_{1}$ & $\mathrm{OO}$ & NN & $\mathrm{C}_{1} \mathrm{C}_{1}$ & ON & $\mathrm{OC}_{1}$ & $\mathrm{NC}_{1}$ & $\mathrm{C}_{1} \mathrm{C}_{4}$ \\
\hline$-\sum_{i} \varepsilon_{s_{i}}$ & 560.02 & 423.34 & 307.59 & 1120.04 & 846.68 & 615.17 & 983.36 & 867.61 & 730.93 & 614.62 \\
\hline $\mathrm{IP}(\triangle \mathrm{SCF})$ & 540.80 & 407.10 & 293.72 & 1174.60 & 895.07 & 654.22 & 951.87 & 841.07 & 705.84 & 593.72 \\
\hline $\operatorname{IP}(\Delta \mathrm{MRCI})$ & 542.57 & 408.38 & 294.93 & 1177.68 & 897.23 & 656.37 & 954.80 & 844.05 & 708.11 & 595.43 \\
\hline Repuls. energy & $\ldots$ & $\ldots$ & $\ldots$ & 128.96 & 112.17 & 95.33 & 2.56 & 10.39 & 3.40 & 5.11 \\
\hline Relax. energy & 19.23 & 16.24 & 13.87 & 74.40 & 63.78 & 56.29 & 34.05 & 36.94 & 28.48 & 26.03 \\
\hline Corr. energy & -1.77 & -1.28 & -1.22 & -3.08 & -2.16 & -2.15 & -2.93 & -2.99 & -2.27 & -1.70 \\
\hline Relat. corr. & 0.48 & 0.27 & 0.14 & 0.98 & 0.55 & 0.28 & 0.75 & 0.62 & 0.41 & 0.28 \\
\hline
\end{tabular}


those computed with the $\triangle \mathrm{SCF}$ and $\triangle \mathrm{MRCI}$ methods, we can estimate the $R\left(S^{-1}\right)$ and $C\left(S^{-1}\right)$ contributions.

The relaxation energies are obtained by subtracting the $\triangle \mathrm{SCF}$ ionization potentials from the corresponding core orbital energies. The correlation energies are the differences between the $\triangle \mathrm{SCF}$ and $\triangle \mathrm{MRCI}$ results. Both the derived relaxation and correlation energies are shown in Table I. As one can see, the relaxation effects play a far more important role than the correlation effects, and their accurate treatment is indispensable when describing core ionization.

The double ionization potential associated with two core vacancies $S_{i}^{-1}$ and $S_{j}^{-1}$ takes on the following appearance:

$$
\begin{aligned}
\operatorname{DIP}\left(S_{i}^{-1}, S_{j}^{-1}\right)= & -\varepsilon_{S_{i}}-\varepsilon_{S_{j}}+E_{R E}\left(S_{i}^{-1}, S_{j}^{-1}\right) \\
& -R\left(S_{i}^{-1}, S_{j}^{-1}\right)-C\left(S_{i}^{-1}, S_{j}^{-1}\right) .
\end{aligned}
$$

The new term $E_{R E}$ which has no analog in Eq. (1) accounts both for electrostatic repulsion between the two core holes and for exchange interaction if these holes occupy different atomic sites. Given that the core holes are well localized each on a different center, the exchange interaction between them is negligibly small and $E_{R E}$ can be described to a good approximation by the inverse of the distance $r_{S_{i} S_{j}}$ between the centers. The repulsion energy of two core holes created on the same atom can be described by the two-electron integral $V_{S_{i} S_{i} S_{i} S_{i}}$.

In Table I, we collect the double ionization potentials of selected states computed with the $\triangle \mathrm{SCF}$ and $\triangle \mathrm{MRCI}$ methods. As in the case of single core ionization, we have estimated the relaxation and correlation energies associated with the removal of two core electrons. According to Eq. (2), $R\left(S_{i}^{-1}, S_{j}^{-1}\right)$ is the difference between the first-order DIP $\left(-\varepsilon_{S_{i}}-\varepsilon_{S_{j}}+E_{R E}\left(S_{i}^{-1}, S_{j}^{-1}\right)\right)$ and the double ionization potential derived with the $\triangle \mathrm{SCF}$ method. As expected, the relaxation effects induced by a double core hole creation are much more pronounced than those induced by a single core hole creation. The correlation contributions $C\left(S_{i}^{-1}, S_{j}^{-1}\right)$ obtained by subtracting the $\triangle \mathrm{MRCI}$ results from the $\triangle \mathrm{SCF}$ ones are, in contrast, of similar size to $C\left(S_{i}^{-1}\right)$ and $C\left(S_{j}^{-1}\right)$.

\section{Relativistic corrections}

A description of core ionization requires relativistic effects to be taken into account. This primarily concerns systems with heavy elements. In systems composed of light atoms, relativistic effects are usually small but not negligible. In the case of p-AP, the relativistic corrections for the single and double IPs vary from 0.14 to $0.98 \mathrm{eV}$ depending on the atomic number and the ionic state (see the last row in Table I). Note that for the light atoms considered here the relativistic correction for a given DIP is the sum of the relativistic corrections for the IPs of the two core holes formed. The same trend was discovered recently in small molecules. ${ }^{23}$

\section{Basis-set convergence of core level IPs and DIPs}

The basis set exerts a noticeable effect on the computed ionization potentials. For demonstration, we carried out

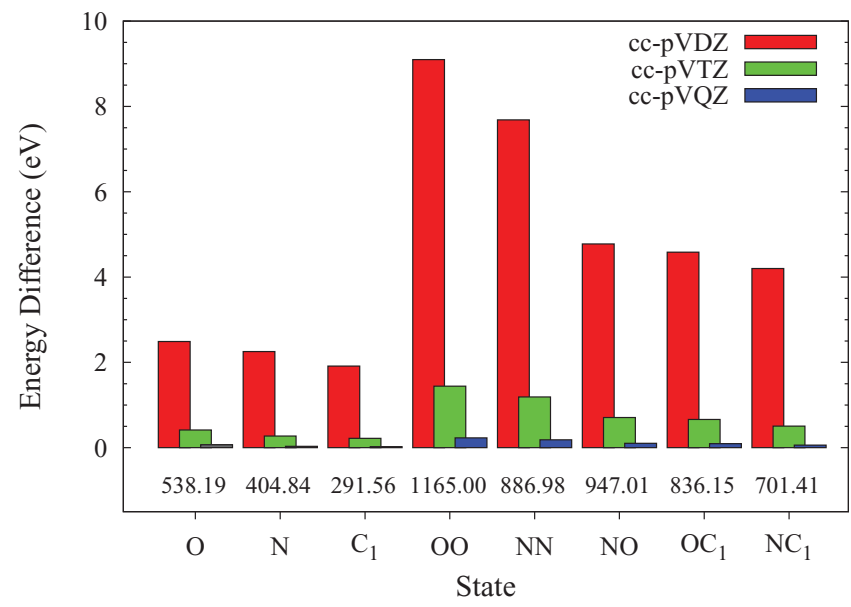

FIG. 3. Energy deviations, from the respective complete-basis-set limits (numbers below boxes), of the $K$-shell single and double ionization potentials of the para-aminophenol molecule computed with different basis sets at the $\triangle \mathrm{SCF}$ level of theory.

$\triangle \mathrm{SCF}$ calculations where basis sets of sizes varying in a broad interval can be easily used, in contrast to post-HartreeFock calculations where a large basis set obviously represents an obstacle. We used the cc-pVXZ (X $=\mathrm{D}, \mathrm{T}, \mathrm{Q})$ basis set suite. $^{25}$ This enabled us not only to get insight into the dependence of the single and double core ionization potentials on the quality of a basis set, but also to establish the completebasis-set (CBS) limits for these potentials by extrapolating the computed results, similar to what has been done for the ground-state SCF and correlation energies. ${ }^{36}$

It turns out that the exponential three-parameter fit

$$
E=a+b \exp (-c X) \text {, }
$$

where $X$ is the cardinal number of the basis set, excellently describes the calculated core level IPs and DIPs. The parameters $a$ corresponding to the CBS limits for selected states are given in Fig. 3. This figure also shows the energy differences between the computed IPs and DIPs and their respective CBS limits. The changes in the IPs and DIPs as a function of the basis set employed are mainly due to changes of the relaxation energies. Since these energies are the biggest in the case of one-site double core ionization (the relaxation energy associated with a one-site DIP is approximately four times larger than that associated with the respective single IP), the corresponding DIPs are subject to the largest changes as a function of the basis-set size (see the OO and NN states in Fig. 3).

Figure 3 clearly demonstrates that making use of the ccpVDZ basis set does not ensure reasonable accuracy of both the single and double ionization potentials. The relaxation energies are significantly underestimated here. Some ionization potentials cannot be well described even with the cc-pVTZ basis set. In contrast, the calculations with the cc-pVQZ basis set provide results which are very close to the CBS limits.

We note that the same applies also for the energy differences between the double and single ionization potentials which are used for deriving the relaxation energies. ${ }^{14,16}$ The above energy differences computed with the cc-pVDZ basis set exhibit significant deviations from the CBS results. On the 
other hand, the relative energies between states of the same type as, for example, between the carbon $1 s$ levels, are much less sensitive to the choice of the basis set. This implies that a reasonably good description of the chemical shifts can be obtained even with a low-quality basis set.

\section{COMPARISON OF INNER-SHELL SINGLE AND DOUBLE IONIZATION POTENTIALS OF THE AMINOPHENOL ISOMERS}

The present section deals with the isomer dependence of the $K$-shell single and double IPs of aminophenol. They have been computed by means of the non-relativistic $\triangle \mathrm{SCF}$ method in conjunction with the cc-pVQZ basis set. The big size of the cc-pVQZ basis set makes the respective MRCI calculations prohibitive. The MRCI calculations are, however, feasible for molecules smaller than aminophenol. In the Appendix, where we assess the accuracy of the $\triangle \mathrm{SCF}$ and $\triangle$ MRCI methods, the calculated DIPs of several small molecules are compared with the experimental values. One can see that correlation effects are relatively small and their neglect still leads to reasonable agreement between the theoretical and experimental results. It is worth mentioning that the results shown in this section are very close to the $\triangle \mathrm{SCF}$ CBS limits of the inner-shell ionization potentials. Due to the excellent account for prevailing relaxation effects, the values calculated here are more reliable than those obtained in Sec. III at the post-Hartree-Fock level of theory using a smaller basis set and where the account for relaxation effects is, in general, deficient.

\section{A. Single ionization potentials}

The $\mathrm{C} 1 s$ ionization potentials of a benzene molecule with its six equivalent carbon atoms are known to be essentially the same. ${ }^{37}$ In substituted benzenes, this "quasidegeneracy" is lifted and an energy splitting between the C $1 s^{-1}$ states thus arises.

In contrast to aminophenol, only one substituent group is present in phenol and aniline and we first address ionization of these two molecules. The corresponding $\mathrm{C} 1 s$ IPs are shown in panels (f) and (g) of Fig. 4. They are also listed in Table S1 of the supplementary material. ${ }^{38}$ In each spectrum, two groups of states can be distinguished as a consequence of distinctive properties of the carbons' neighbors. The highenergy group contains only one state describing ionization of the carbon atom adjacent to $\mathrm{OH}$ and $\mathrm{NH}_{2}$ in phenol and aniline, respectively. The hydroxyl and amine groups withdraw electron density from the neighbors making the latter positively charged and their core level IPs larger. The corresponding energy shift with respect to the single IP of benzene calculated at the $\triangle \mathrm{SCF} / \mathrm{cc}-\mathrm{pVQZ}$ level of theory $(290.34 \mathrm{eV}$, grey dotted vertical line in Fig. 4) is $1.08 \mathrm{eV}$ in aniline and 1.81 $\mathrm{eV}$ in phenol. For comparison, the chemical shift associated with the carbon atom adjacent to fluorine in fluorobenzene is $2.44 \mathrm{eV}^{39}$ These chemical shifts correlate with the electronegativity along the series $\mathrm{NH}_{2}<\mathrm{OH}<\mathrm{F}$.

The states associated with the carbons adjacent to hydrogen atoms form the second group. While in fluorobenzene, all

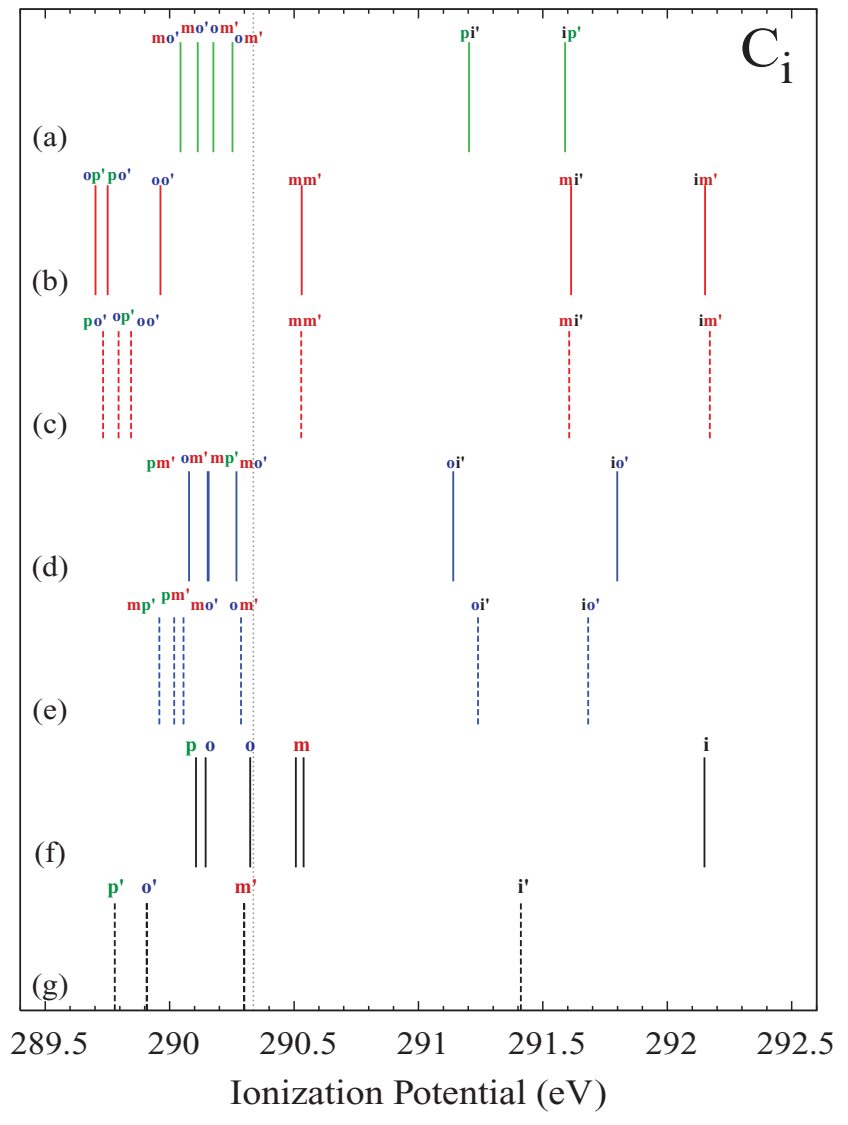

FIG. 4. Calculated ( $\triangle \mathrm{SCF} / \mathrm{cc}-\mathrm{pVQZ}) K$-shell single ionization potentials of the carbon atoms in the p-AP (a), m-AP(A, B) ((b) and (c), respectively), o$\mathrm{AP}(\mathrm{A}, \mathrm{B})((\mathrm{d})$ and (e), respectively) molecules as well as in phenol (f), aniline $(\mathrm{g})$, and benzene (grey dotted line). The letters above the lines describe the positions of the hydroxyl (without the prime) and amine groups (with the prime) relative to the core ionized carbon atom: para (p), meta (m), ortho (o), and ipso (i).

these states exhibit positive chemical shifts, ${ }^{39}$ in phenol only two states from this group have IPs larger than those of benzene and none of them has positive chemical shift in aniline which is in accord with the electron withdrawing power of the corresponding substituents. Note that the carbon atoms in the para and ortho positions relative to the site of ionization have lower IPs (the energy lowering is stronger in the para case) than the carbons in the meta positions due to resonance effects. The substituents act as $\pi$-electron donors for the para and ortho carbons but not for the meta carbons. It is also worth pointing out the differences in the IPs (particularly strong in the ortho case) of the carbon atoms located on the same and opposite site relative to the position of the hydrogen atom in the hydroxyl group. The former carbons lose more electron density through interacting with the dipole of the $\mathrm{COH}$ group and their IPs are thus larger.

The $\mathrm{OH}$ and $\mathrm{NH}_{2}$ groups are present simultaneously in the aminophenol isomers. The C $1 s$ IPs of these compounds are shown in panels (a)-(e) of Fig. 4 and also given in Table II. Often the effect of multiple substituents, e.g., on the chemical shift, is described in terms of an additivity model ${ }^{39}$ where the total effect of the substituents is represented as a sum of independent effects of the individual substituents. We did not inspect thoroughly the performance of this model 
TABLE II. $K$-shell single ionization potentials of five different aminophenol isomers calculated at the $\triangle \mathrm{SCF} / \mathrm{cc}$-pVQZ level of theory. The corresponding orbital $(-\varepsilon)$ and relaxation $(\mathrm{R})$ energies are shown as well. All values are in $\mathrm{eV}$. The carbon atoms are numerated in a clockwise manner starting from $\mathrm{C}_{1}$ adjacent to the $\mathrm{OH}$ group in all molecules. $\mathrm{C}_{2}, \mathrm{C}_{3}$, and $\mathrm{C}_{4}$ are adjacent to the $\mathrm{NH}_{2}$ group in o-AP, m-AP, and p-AP, respectively.

\begin{tabular}{|c|c|c|c|c|}
\hline System & Center & $-\varepsilon$ & $\mathrm{R}$ & IP \\
\hline \multirow[t]{8}{*}{ p-AP } & $\mathrm{O}$ & 560.88 & 22.62 & 538.26 \\
\hline & $\mathrm{N}$ & 423.94 & 19.07 & 404.87 \\
\hline & $\mathrm{C}_{1}$ & 307.62 & 16.03 & 291.59 \\
\hline & $\mathrm{C}_{2}$ & 306.23 & 15.98 & 290.25 \\
\hline & $\mathrm{C}_{3}$ & 306.08 & 16.04 & 290.04 \\
\hline & $\mathrm{C}_{4}$ & 307.16 & 15.96 & 291.20 \\
\hline & $\mathrm{C}_{5}$ & 306.14 & 16.03 & 290.11 \\
\hline & $\mathrm{C}_{6}$ & 306.17 & 15.99 & 290.18 \\
\hline \multirow[t]{8}{*}{ m-AP(A) } & $\mathrm{O}$ & 560.20 & 21.72 & 538.48 \\
\hline & $\mathrm{N}$ & 423.63 & 18.48 & 405.15 \\
\hline & $\mathrm{C}_{1}$ & 307.73 & 15.58 & 292.15 \\
\hline & $\mathrm{C}_{2}$ & 305.52 & 15.56 & 289.96 \\
\hline & $\mathrm{C}_{3}$ & 307.16 & 15.55 & 291.61 \\
\hline & $\mathrm{C}_{4}$ & 305.38 & 15.63 & 289.75 \\
\hline & $\mathrm{C}_{5}$ & 306.09 & 15.56 & 290.53 \\
\hline & $\mathrm{C}_{6}$ & 305.33 & 15.63 & 289.70 \\
\hline \multirow[t]{8}{*}{ m-AP(B) } & $\mathrm{O}$ & 560.22 & 21.73 & 538.49 \\
\hline & $\mathrm{N}$ & 423.58 & 18.48 & 405.10 \\
\hline & $\mathrm{C}_{1}$ & 307.74 & 15.57 & 292.17 \\
\hline & $\mathrm{C}_{2}$ & 305.41 & 15.56 & 289.85 \\
\hline & $\mathrm{C}_{3}$ & 307.15 & 15.54 & 291.61 \\
\hline & $\mathrm{C}_{4}$ & 305.36 & 15.63 & 289.73 \\
\hline & $\mathrm{C}_{5}$ & 306.07 & 15.54 & 290.53 \\
\hline & $\mathrm{C}_{6}$ & 305.43 & 15.63 & 289.80 \\
\hline \multirow[t]{8}{*}{ o-AP(A) } & $\mathrm{O}$ & 560.03 & 21.76 & 538.27 \\
\hline & $\mathrm{N}$ & 423.74 & 18.43 & 405.31 \\
\hline & $\mathrm{C}_{1}$ & 307.46 & 15.66 & 291.80 \\
\hline & $\mathrm{C}_{2}$ & 306.81 & 15.67 & 291.14 \\
\hline & $\mathrm{C}_{3}$ & 305.88 & 15.61 & 290.27 \\
\hline & $\mathrm{C}_{4}$ & 305.69 & 15.61 & 290.08 \\
\hline & $\mathrm{C}_{5}$ & 305.79 & 15.63 & 290.16 \\
\hline & $\mathrm{C}_{6}$ & 305.73 & 15.58 & 290.15 \\
\hline \multirow[t]{8}{*}{ o-AP(B) } & $\mathrm{O}$ & 560.24 & 21.72 & 538.52 \\
\hline & $\mathrm{N}$ & 423.32 & 18.48 & 404.84 \\
\hline & $\mathrm{C}_{1}$ & 307.41 & 15.73 & 291.68 \\
\hline & $\mathrm{C}_{2}$ & 306.85 & 15.61 & 291.24 \\
\hline & $\mathrm{C}_{3}$ & 305.72 & 15.66 & 290.06 \\
\hline & $\mathrm{C}_{4}$ & 305.69 & 15.67 & 290.02 \\
\hline & $\mathrm{C}_{5}$ & 305.65 & 15.69 & 289.96 \\
\hline & $\mathrm{C}_{6}$ & 305.87 & 15.58 & 290.29 \\
\hline
\end{tabular}

in the case of aminophenol since this is beyond the scope of the present paper. Only several IPs in different isomers were analyzed instead and only by using the linear additivity model. In general, the model performs well in all but the o-AP molecules. The chemical shifts of the two states marked by oi' and io' in the spectrum the o-AP(A) isomer (see Fig. 4(e)) are particularly poorly reproduced by the additivity model. This can be explained by the substantial geometry changes in the ortho-aminophenol molecules compared to the geometries of the phenol and aniline molecules.
It is apparent that distinguishing the aminophenol isomers on the basis of their $\mathrm{C} 1 s$ IPs is a rather challenging task. A peculiarity is found only in the spectrum of m-AP where the low-energy group of states exhibits a pronounced energy splitting.

Let us now look at the $\mathrm{N} 1 s$ and $\mathrm{O} 1 s$ ionization potentials of aminophenol. Their isomer dependence is shown in Fig. 5(a) (see also Table II). As one can see, this dependence is also rather weak. The ionization potentials differ by a maximum of only $0.26 \mathrm{eV}$ in the case of oxygen ionization and by a maximum of $0.47 \mathrm{eV}$ when nitrogen is ionized. The largest effect is exerted by the relative orientation of the hydroxyl and amine groups in the o-AP molecule. We note that p-AP has lower IPs than $\mathrm{m}$-AP which is in accord with the resonance effects rules. The IPs of the ortho-aminophenols do not strictly obey these rules. This might be due to the remarkably different geometries of these systems and, specifically in the case of o-AP(A), due to a formation of an intramolecular hydrogen bond between the $\mathrm{OH}$ and $\mathrm{NH}_{2}$ groups.

\section{B. Double ionization potentials}

The double ionization potentials associated with two core vacancies created on the same atom bring little additional insight compared to the respective single ionization potentials. The main difference between these two types of potentials, apart from their absolute values, is that the energy separations in the manifold of the one-site DIPs are about 2-2.5 times larger than the energy separations in the manifold of the single IPs (see Fig. 5). A nonuniform increase of the energy separations may originate due to different repulsion energies between the two core holes as well as due to different relaxation effects induced by double core ionization in distinct aminophenol isomers. This can be inferred from the following representation of the one-site DIP derived from Eq. (2):

$$
\operatorname{DIP}\left(S^{-1}, S^{-1}\right)=2 \times \operatorname{IP}\left(S^{-1}\right)+V_{S S S S}-E R\left(S^{-1}, S^{-1}\right) .
$$

Note that the $C\left(S^{-1}, S^{-1}\right)$ term is omitted since we are dealing with the $\triangle \mathrm{SCF}$ results. It turns out that the repulsion energies described by $V_{S S S S}$ are affected neither by the mutual arrangement nor by the relative orientation of the $\mathrm{OH}$ and $\mathrm{NH}_{2}$ groups. In contrast, $E R\left(S^{-1}, S^{-1}\right)$ called the excess relaxation energy ${ }^{23}$ (known also as static relaxation ${ }^{8}$ ) varies slightly from one isomer to another (see Table III). As follows from its name, the excess relaxation energy describes non-additive relaxation effects originating upon creation of multiple core vacancies on the same atomic center. $E R\left(S^{-1}, S^{-1}\right)$ alone is nearly twice as large as the relaxation energy $R\left(S^{-1}\right)$ of a single core vacancy $S^{-1}$, and the total relaxation effects induced by one-site double ionization are thus about four times larger than the relaxation effects induced by single core ionization, being approximately quadratic in the hole charge.

Let us now consider two-site double ionization potentials of aminophenol. Those related to the N/O double core holes 

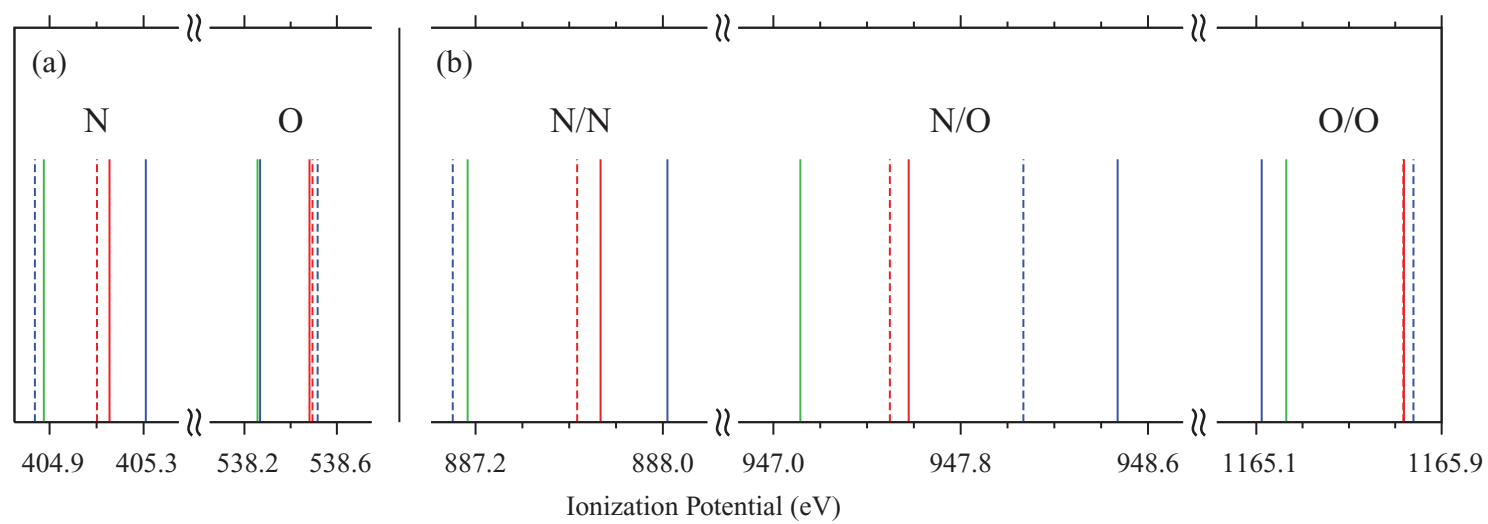

FIG. 5. Calculated ( $\triangle \mathrm{SCF} / \mathrm{cc}-\mathrm{pVQZ}$ ) $K$-shell single (a) and double (b) ionization potentials related to the N $1 s$ and $\mathrm{O} 1 s$ vacancies in the aminophenol isomers: p-AP (green solid lines), m-AP(A,B) (red solid and dashed lines, respectively), and o-AP(A,B) (blue solid and dashed lines, respectively).

are presented in Fig. 5(b). The state ordering in this manifold expectedly reflects the internuclear separations between the core ionized atoms in the aminophenol isomers: the greater the $\mathrm{N}-\mathrm{O}$ distance, the smaller is the respective DIP. Note, however, that although the repulsion energies between $\mathrm{O} 1 \mathrm{~s}$ and $\mathrm{N} 1 s$ holes in p-AP and o-AP(A) differ by $2.57 \mathrm{eV}$, the difference between the respective DIPs is only $1.35 \mathrm{eV}$ (see Table III).

The calculated two-site double ionization potentials $\mathrm{N} / \mathrm{C}_{i}$ and $\mathrm{O} / \mathrm{C}_{i}$ for all aminophenol isomers are shown in Figs. 6 and 7, respectively. The carbon atoms are numerated in a clockwise manner starting from the atom adjacent to the $\mathrm{OH}$ group. The carbon atoms adjacent to the amine group have the index numbers 2,3 , and 4 in ortho-, meta-, and paraaminophenols, respectively. One expects that for each individual molecule, the $\mathrm{N} / \mathrm{C}_{i}$ and $\mathrm{O} / \mathrm{C}_{i}$ DIPs reflect differences in the $\mathrm{N}_{-} \mathrm{C}_{i}$ and $\mathrm{O}-\mathrm{C}_{i}$ distances. However, this is often not the case. For example, the N/C $\mathrm{C}_{1}$ DIP in p-AP, despite the largest $\mathrm{N}-\mathrm{C}_{1}$ distance, is not the smallest one in the manifold of the $\mathrm{N} / \mathrm{C}_{i}$ potentials. One of the reasons has to do with the single IP of the $\mathrm{C}_{1} 1 s$ vacancy which is the largest among the car-

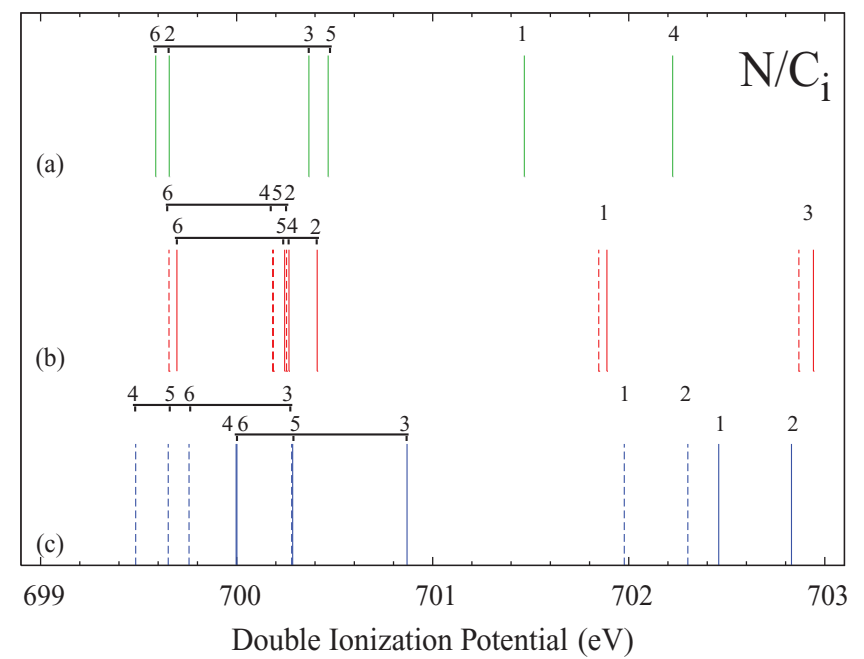

FIG. 6. Calculated ( $\triangle \mathrm{SCF} / \mathrm{cc}-\mathrm{pVQZ}) K$-shell two-site double ionization potentials N/C of p-AP (a), m-AP (b), and o-AP (c). The vertical solid and dashed lines represent the energies of the A and B isomers, respectively. The carbon atoms are numerated as described in the caption of Table II. bon atoms and this overcompensates the effect of the small electrostatic repulsion. We also draw attention to the $\mathrm{N} / \mathrm{C}_{4}$ and $\mathrm{N} / \mathrm{C}_{6}$ DIPs in o-AP(B) and to the $\mathrm{O} / \mathrm{C}_{2}$ and $\mathrm{O} / \mathrm{C}_{6}$ DIPs in $\mathrm{m}$-AP(A) which reveal noticeable differences although the interatomic distances involved are nearly identical.

The following equation provides insight into what influences the sensitivity of two-site DIPs to the chemical environment:

$$
\begin{aligned}
\operatorname{DIP}\left(S_{i}^{-1}, S_{j}^{-1}\right)= & \operatorname{IP}\left(S_{i}^{-1}\right)+\operatorname{IP}\left(S_{j}^{-1}\right) \\
& +1 / r_{S_{i} S_{j}}-\operatorname{IR}\left(S_{i}^{-1}, S_{j}^{-1}\right) .
\end{aligned}
$$

Together with the repulsion energy and the ionization potentials of single core vacancies, the interatomic relaxation energy $\operatorname{IR}\left(S_{i}^{-1}, S_{j}^{-1}\right)($ Ref. 23) plays an important role in a two-site DIP (correlation effects are not considered here). $\operatorname{IR}\left(S_{i}^{-1}, S_{j}^{-1}\right)$ describes non-additive relaxation effects originating upon creation of multiple core vacancies on different atomic centers including a deviation of the repulsion energy from the $1 / r_{S_{i} S_{j}}$ dependence due to, e.g., screening of the holes. ${ }^{17}$

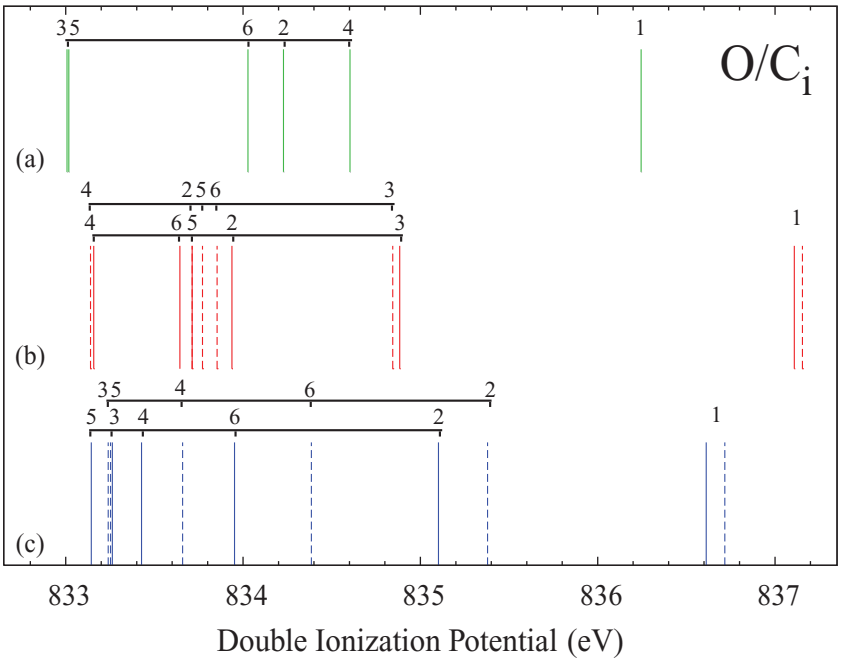

FIG. 7. Calculated ( $\triangle \mathrm{SCF} / \mathrm{cc}-\mathrm{pVQZ}) K$-shell two-site double ionization potentials O/C of p-AP (a), m-AP (b), and o-AP (c). The vertical solid and dashed lines represent the energies of the A and $\mathrm{B}$ isomers, respectively. The carbon atoms are numerated as described in the caption of Table II. 
TABLE III. Selected $K$-shell double ionization potentials of the five different aminophenol isomers together with the excess (ER, underlined values) and interatomic (IR) relaxation energies calculated at the $\triangle \mathrm{SCF} / \mathrm{cc}-\mathrm{pVQZ}$ level of theory. All values are in eV. The carbon atoms are numerated as described in the caption of Table II.

\begin{tabular}{|c|c|c|c|c|c|}
\hline \multirow{3}{*}{$\begin{array}{l}\text { System } \\
\text { p-AP }\end{array}$} & \multirow{3}{*}{$\frac{\text { Center }}{\mathrm{O}}$} & \multirow{2}{*}{\multicolumn{2}{|c|}{$\begin{array}{l}\text { DIP } \\
\qquad \\
\end{array}$}} & \multirow{2}{*}{ DIP } & \multirow[t]{2}{*}{ IR/ER } \\
\hline & & & & & \\
\hline & & 1165.23 & 40.26 & 947.12 & -1.42 \\
\hline & $\mathrm{N}$ & 947.12 & -1.42 & 887.17 & 34.75 \\
\hline & $\mathrm{C}_{1}$ & 836.25 & 4.00 & 701.47 & -1.61 \\
\hline & $\mathrm{C}_{2}$ & 834.23 & 0.22 & 699.66 & -0.67 \\
\hline & $\mathrm{C}_{3}$ & 833.01 & -0.82 & 700.37 & 0.44 \\
\hline & $\mathrm{C}_{4}$ & 834.60 & -1.72 & 702.22 & 3.99 \\
\hline & $\mathrm{C}_{5}$ & 833.02 & -0.76 & 700.47 & 0.40 \\
\hline & $\mathrm{C}_{6}$ & 834.03 & 0.37 & 699.59 & -0.69 \\
\hline \multirow[t]{8}{*}{ m-AP(A) } & $\mathrm{O}$ & 1165.74 & $\underline{40.20}$ & 947.58 & -0.97 \\
\hline & $\mathrm{N}$ & 947.58 & -0.97 & 887.73 & 34.74 \\
\hline & $\mathrm{C}_{1}$ & 837.11 & 3.94 & 701.89 & -0.71 \\
\hline & $\mathrm{C}_{2}$ & 833.94 & 0.47 & 700.41 & 0.61 \\
\hline & $\mathrm{C}_{3}$ & 834.88 & -0.89 & 702.94 & 4.01 \\
\hline & $\mathrm{C}_{4}$ & 833.16 & -1.49 & 700.27 & 0.53 \\
\hline & $\mathrm{C}_{5}$ & 833.71 & -0.80 & 700.24 & -0.70 \\
\hline & $\mathrm{C}_{6}$ & 833.64 & 0.52 & 699.70 & -1.44 \\
\hline \multirow[t]{8}{*}{$\mathrm{m}-\mathrm{AP}(\mathrm{B})$} & $\mathrm{O}$ & 1165.73 & 40.23 & 947.50 & -0.93 \\
\hline & $\mathrm{N}$ & 947.50 & -0.93 & 887.63 & $\underline{34.74}$ \\
\hline & $\mathrm{C}_{1}$ & 837.15 & 3.94 & 701.85 & -0.71 \\
\hline & $\mathrm{C}_{2}$ & 833.71 & 0.60 & 700.25 & 0.59 \\
\hline & $\mathrm{C}_{3}$ & 834.84 & -0.85 & 702.87 & 4.04 \\
\hline & $\mathrm{C}_{4}$ & 833.14 & -1.48 & 700.18 & 0.54 \\
\hline & $\mathrm{C}_{5}$ & 833.77 & -0.86 & 700.19 & -0.69 \\
\hline & $\mathrm{C}_{6}$ & 833.85 & 0.39 & 699.73 & -1.43 \\
\hline \multirow[t]{8}{*}{ o-AP(A) } & $\mathrm{O}$ & 1165.12 & $\underline{40.38}$ & 948.47 & 0.22 \\
\hline & $\mathrm{N}$ & 948.47 & 0.22 & 888.02 & 34.77 \\
\hline & $\mathrm{C}_{1}$ & 836.61 & 3.90 & 702.46 & 0.50 \\
\hline & $\mathrm{C}_{2}$ & 835.10 & 0.27 & 702.83 & 3.67 \\
\hline & $\mathrm{C}_{3}$ & 833.26 & -0.83 & 700.87 & 0.56 \\
\hline & $\mathrm{C}_{4}$ & 833.43 & -1.65 & 700.00 & -0.77 \\
\hline & $\mathrm{C}_{5}$ & 833.14 & -0.82 & 700.29 & -1.43 \\
\hline & $\mathrm{C}_{6}$ & 833.95 & 0.44 & 700.00 & -0.70 \\
\hline \multirow[t]{8}{*}{ o-AP(B) } & $\mathrm{O}$ & 1165.78 & $\underline{40.23}$ & 948.07 & 0.42 \\
\hline & $\mathrm{N}$ & 948.07 & 0.42 & 887.10 & 34.74 \\
\hline & $\mathrm{C}_{1}$ & 836.71 & 3.88 & 701.98 & 0.44 \\
\hline & $\mathrm{C}_{2}$ & 835.38 & 0.33 & 702.30 & 3.97 \\
\hline & $\mathrm{C}_{3}$ & 833.24 & -0.78 & 700.28 & 0.50 \\
\hline & $\mathrm{C}_{4}$ & 833.66 & -1.66 & 699.48 & -0.73 \\
\hline & $\mathrm{C}_{5}$ & 833.25 & -0.89 & 699.76 & -1.56 \\
\hline & $\mathrm{C}_{6}$ & 834.39 & 0.37 & 699.65 & -0.66 \\
\hline
\end{tabular}

An analysis of the N/O DIPs shows that their reduced sensitivity to the chemical environment may be traced to the interatomic relaxation energy. As seen from Table III, the latter varies substantially from one isomer to another. In each $\mathrm{o}-\mathrm{AP}$ molecule where the oxygen and nitrogen atoms reside on one side, $\operatorname{IR}\left(\mathrm{O} 1 s^{-1}, \mathrm{~N} 1 s^{-1}\right)$ is positive and the respective DIP is thus lowered. Here the environment supplies the ionized oxygen and nitrogen atoms, which work cooperatively, with the electron density that enhances the overall relaxation effects. In contrast, in the para-aminophenol molecule $\mathrm{O}$ and $\mathrm{N}$ locate on the opposite molecular sides and the overall re-

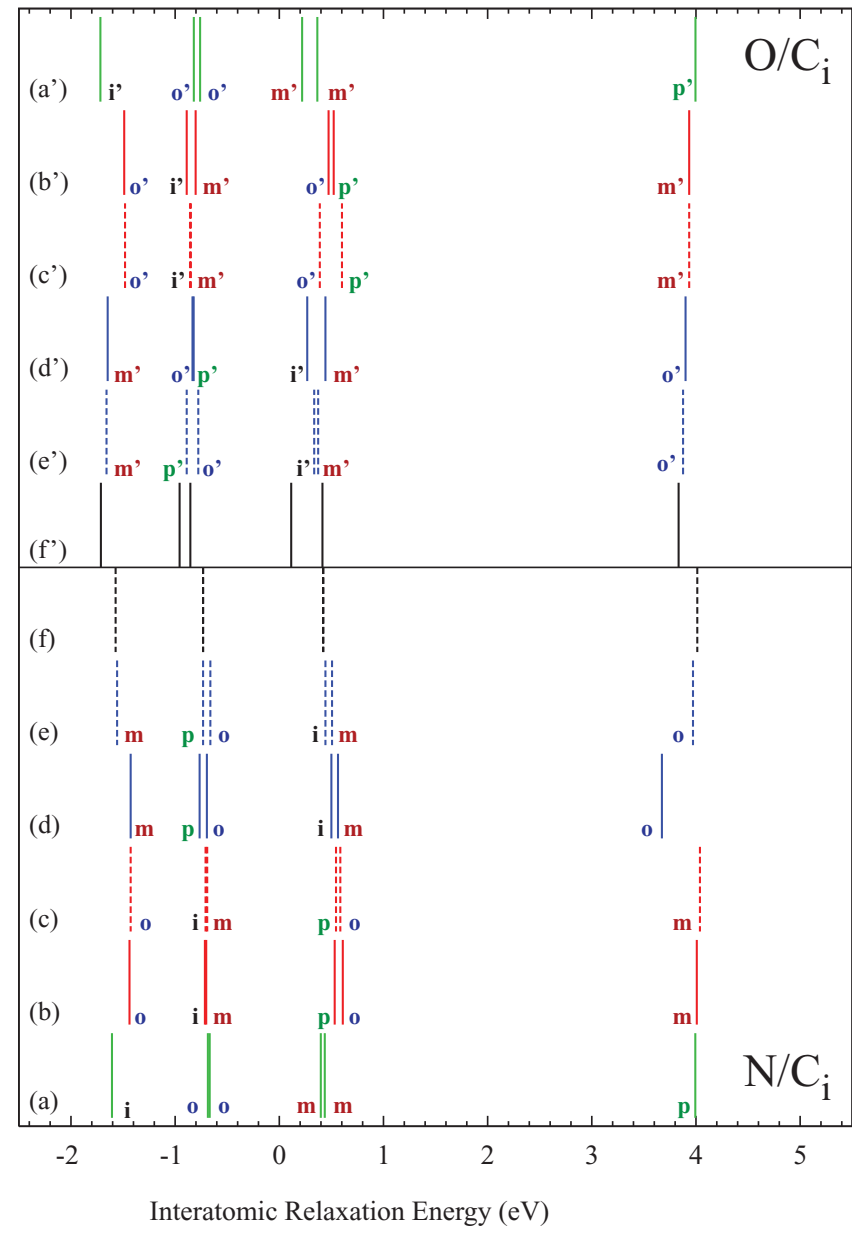

FIG. 8. Interatomic relaxation energies associated with the two-site double ionization potentials $\mathrm{O} / \mathrm{C}$ and N/C in the aminophenol (a-e, $\left.\mathrm{a}^{\prime}-\mathrm{e}^{\prime}\right)$, phenol $\left(\mathrm{f}^{\prime}\right)$ and aniline (f) molecules. The letters alongside of the lines point out on the position of the second, neutral, substituent group relative to the core ionized carbon atom (see the caption of Fig. 4 for designations).

laxation effect is smaller than expected due to the negative interatomic relaxation energy $\operatorname{IR}\left(\mathrm{O} 1 s^{-1}, \mathrm{~N} 1 s^{-1}\right)$. A part of the electron density of the atoms between $\mathrm{N}$ and $\mathrm{O}$, instead of flowing completely to one ionized site as in the case of a single core hole, has to be shared now between two ionized sites. ${ }^{17}$ The respective DIPs thus increase.

The same situation is found in the case of $\mathrm{N} / \mathrm{C}_{i}$ and $\mathrm{O} / \mathrm{C}_{i}$ double ionization. The corresponding interatomic relaxation energies are represented as the vertical lines in Fig. 8 for all the five aminophenol isomers as well as for the phenol and aniline molecules. Four groups of states are distinguished in each spectrum according to the mutual positions of the core ionized substituent group and carbon atom. In the energy decreasing order, these states correspond to the ipso (i) ortho (o), meta (m), and para (p) positions. The letter shown alongside of each line in the spectra of the aminophenols indicate the position of the second, neutral, substituent group relative to the carbon atom with the core vacancy. When the two core holes are created in the adjacent atoms (the ipso position), they work together to polarize the rest of the molecule and the relaxation energy is by about $4 \mathrm{eV}$ larger than the sum of the relaxation energies associated with the individual core holes. As soon as the two holes move apart, their cooperation 
TABLE IV. Comparison of the DIPs (in eV) calculated with different methods and basis sets with the experimental values for small closed-shell molecules. The experimental results were taken from Refs. 6, 14, 15.

\begin{tabular}{|c|c|c|c|c|c|c|}
\hline \multirow[b]{2}{*}{ Molecule } & \multirow[b]{2}{*}{ State } & \multicolumn{2}{|c|}{$\Delta \mathrm{SCF}$} & \multicolumn{2}{|c|}{$\Delta \mathrm{MRCI}$} & \multirow[b]{2}{*}{ Expt. } \\
\hline & & cc-pVQZ & cc-pCVTZ & cc-pVQZ & cc-pCVTZ & \\
\hline $\mathrm{CO}$ & $\mathrm{O} 1 s^{-2}$ & 1175.40 & 1175.49 & 1177.02 & 1177.90 & $1178.0 \pm 0.8$ \\
\hline $\mathrm{NH}_{3}$ & $\mathrm{~N} 1 s^{-2}$ & 891.28 & 891.34 & 891.98 & 892.50 & $892.0 \pm 0.5$ \\
\hline $\mathrm{CH}_{4}$ & $\mathrm{C} 1 s^{-2}$ & 650.92 & 651.01 & 650.59 & 651.56 & $651.5 \pm 0.5$ \\
\hline \multirow[t]{2}{*}{$\mathrm{N}_{2}$} & $\mathrm{~N} 1 s^{-2}$ & 901.62 & 901.65 & 901.71 & 902.51 & $902.6 \pm 0.5$ \\
\hline & $\mathrm{N} 1 s^{-1} \mathrm{~N} 1 s^{-1}$ & 838.18 & 838.07 & 834.86 & 835.96 & 835.9 \\
\hline
\end{tabular}

weakens rapidly. As one can see, in the ortho position the gain in the relaxation energy does not exceed $0.6 \mathrm{eV}$. The holes start to work against each other when they are in the meta and para positions. The environment between the holes is depolarized, especially in the latter case, compared to the situation when only a single core hole is created (see also Ref. 17). The interatomic relaxation energy thus gets negative.

By comparing the interatomic relaxation energies of aminophenols with those of phenol and aniline, one sees that with a few exceptions the effect of the second substituent is rather small. In the majority of cases, the presence of another substituent increases the interatomic relaxation energy. Likely, due to the resonance effects, the energy increases are usually larger when the neutral substituent group is in the para and ortho positions than in the meta and ipso ones relative to the core ionized carbon atom.

The above trends seem to hold even when correlation effects are taken into account. In the absence of $\triangle \mathrm{MRCI} / \mathrm{cc}-$ pVQZ results, we draw this conclusion on the basis of the $\triangle \mathrm{MRCI} / 6-31 \mathrm{G}^{*}$ data from Sec. III obtained for all five aminophenol isomers. We found that for the atoms and atomic pairs from Table I, the orderings of the related IPs and DIPs remain unchanged upon adding the correlation contributions, although the energy spacings do change. We do not exclude the possibility, however, that some close lying potentials, in particular those with a vacancy on a carbon atom, do interchange their positions in the spectra in response to electron correlation.

\section{SUMMARY AND CONCLUSIONS}

The double core ionization potentials, especially those related to states with two core vacancies on different atomic centers, are more sensitive to the chemical environment than are the single core ionization potentials, and the present exploration of the para-, meta-, and ortho-aminophenol molecules has provided additional support for this fact. Structural differences between distinctive isomers are an obvious reason for the enhanced sensitivity of the two-site DIPs. Our study has demonstrated that, in addition, electronic relaxation and, to a much lesser extent, correlation accompanying double ionization lie at the heart of this phenomenon.

The relaxation energy constitutes a substantial part of core level single and double ionization potentials and this should be taken into account when choosing the computa- tional method and the basis set. For single core levels, the $\triangle \mathrm{SCF}$ method is known to perform well in treating relaxation effects. The comparison of the experimental DIPs with the respective $\triangle \mathrm{SCF}$ results for several small molecules made in the Appendix has shown that $\triangle \mathrm{SCF}$ works well also in the case of double core ionization. The amount of the relaxation energy accounted for in computations and thus the quality of the calculated single and double ionization potentials depends, however, significantly on the basis set used. In most cases, the cc-pVTZ basis set already leads to reasonable results. The $\triangle \mathrm{SCF} / \mathrm{cc}-\mathrm{pVQZ}$ results for single and double IPs are in excellent agreement with the respective complete-basisset limits.

The relaxation energy is not an additive quantity. A creation of a second core hole on the same atom, for example, enhances the relaxation energy approximately four times. Deviations from additivity of the relaxation energy are not as dramatic in the case of two-site double ionization. In spite of their smaller magnitudes, these effects, however, significantly influence the sensitivity of the two-site double ionization potentials to the chemical environment. Importantly, the nonadditivity effects depend strongly on the positions of the core ionized atoms relative to each other in the molecule.

Relativistic effects play a minor role for the light atoms present in the aminophenol molecule. Interestingly, the relativistic corrections for inner-shell double ionization potentials turn out to be additive.

Correlation effects are taken into account in the paper at the singles-and-doubles $\triangle \mathrm{MRCI}$ level of theory. According to our calculations performed for some small molecules (see the Appendix), a combination of this method with a suitable basis set is able to provide a reliable description of inner-shell DIPs. As $\triangle$ MRCI calculations with the cc-pVQZ basis set are not feasible in the case of aminophenol, we got fair estimates of the correlation energies by utilizing the relatively small 6$31 \mathrm{G}^{*}$ basis set. The correlation energies are small and their isomer dependence is not well pronounced in comparison to the relaxation energies.

\section{ACKNOWLEDGMENTS}

We would like to thank V. P. Vysotskiy for extracting two-electron integrals from $a b$ initio data. We also thank one of the numerous referees of our paper for his suggestions which have led to Fig. 8. N.V.K. and L.S.C. gratefully acknowledge financial support by the DFG. 


\section{APPENDIX: ASSESSMENT OF THE ACCURACY OF THE $\triangle$ SCF AND $\triangle M R C I$ METHODS FOR THE DOUBLE CORE IONIZATION POTENTIALS}

The utility of the $\triangle \mathrm{SCF}$ and $\triangle \mathrm{MRCI}$ methods in dealing with single core IPs has been well demonstrated in the literature. The recent appearance of the experimental results on molecular double core holes ${ }^{6,14,15}$ enables us to assess the accuracy of these methods with respect to the corresponding DIPs. In Table IV, we compare the calculated $K$-shell DIPs with the measured ones for several small molecules. The calculations were performed by means of the $\triangle \mathrm{SCF}$ and $\triangle \mathrm{MRCI}$ procedures with the relativistic corrections taken into account through the ZORA formalism. We used the cc-pVQZ and ccpCVTZ basis sets.

Except for the two-site DIP of $\mathrm{N}_{2}$ where the experimental value has been defined with a rather big uncertainty ${ }^{6}$ and the one-site DIP of CO, the $\triangle \mathrm{SCF} / \mathrm{cc}-\mathrm{pVQZ}$ results show satisfactory agreement with the experimental results: the energy differences constitute less than $1 \mathrm{eV}$. The account for correlation effects via $\triangle \mathrm{MRCI}$ improves the quality of the theoretical results especially in the two problematic cases. As one can also see from Table IV, excellent agreement with the experiment is achieved when the $\triangle \mathrm{MRCI}$ method is used in conjunction with the cc-pCVTZ basis set. This is apparently related to the peculiarity of the cc-pCVTZ basis set. It contains tight functions and was specially designed for a better description of the core-core and core-valence correlation effects. $^{40}$

${ }^{1}$ R. Santra, N. V. Kryzhevoi, and L. S. Cederbaum, Phys. Rev. Lett. 103 013002 (2009).

${ }^{2}$ J. Arthur, P. Anfinrud, P. Audebert, K. Bane, I. Ben-Zvi, V. Bharadwaj, R. Bionta, P. Bolton, M. Borland, P. H. Bucksbaum, R. C. Cauble, J. Clendenin, M. Cornacchia, G. Decker, P. Den Hartog, S. Dierker, D. Dowell, D. Dungan, P. Emma, I. Evans, G. Faigel, R. Falcone, W. M. Fawley, M. Ferrario, A. S. Fisher, R. R. Freeman, J. Frisch, J. Galayda, J.-C. Gauthier, S. Gierman, E. Gluskin, W. Graves, J. Hajdu, J. Hastings, K. Hodgson, Z. Huang, R. Humphry, P. Ilinski, D. Imre, C. Jacobsen, C.-C. Kao, K. R. Kase, K.-J. Kim, R. Kirby, J. Kirz, L. Klaisner, P. Krejcik, K. Kulander, O. L. Landen, R. W. Lee, C. Lewis, C. Limborg, E. I. Lindau, A. Lumpkin, G. Materlik, S. Mao, J. Miao, S. Mochrie, E. Moog, S. Milton, G. Mulhollan, K. Nelson, W. R. Nelson, R. Neutze, A. Ng, D. Nguyen, H.-D. Nuhn, D. T. Palmer, J. M. Paterson, C. Pellegrini, S. Reiche, M. Renner, D. Riley, C. V. Robinson, S. H. Rokni, S. J. Rose, J. Rosenzweig, R. Ruland, G. Ruocco, D. Saenz, S. Sasaki, D. Sayre, J. Schmerge, D. Schneider, C. Schroeder, L. Serafini, F. Sette, S. Sinha, D. van der Spoel, B. Stephenson, G. Stupakov, M. Sutton, A. Szöke, R. Tatchyn, A. Toor, E. Trakhtenberg, I. Vasserman, N. Vinokurov, X. J. Wang, D. Waltz, J. S. Wark, E. Weckert, Wilson-Squire Group, H. Winick, M. Woodley, A. Wootton, M. Wulff, M. Xie, R. Yotam, L. Young, and A. Zewail, "Linac coherent light source (LCLS): Conceptual design report," Report No. SLAC-R-593 and UC-414, 2002.

${ }^{3}$ P. Emma, R. Akre, J. Arthur, R. Bionta, C. Bostedt, J. Bozek, A. Brachmann, P. Bucksbaum, R. Coffee, F.-J. Decker, Y. Ding, D. Dowell, S. Edstrom, A. Fisher, J. Frisch, S. Gilevich, J. Hastings, G. Hays, Ph. Hering, Z. Huang, R. Iverson, H. Loos, M. Messerschmidt, A. Miahnahri, S. Moeller, H.-D. Nuhn, G. Pile, D. Ratner, J. Rzepiela, D. Schultz, T. Smith, P. Stefan, H. Tompkins, J. Turner, J. Welch, W. White, J. Wu, G. Yocky, and J. Galayda, Nat. Photonics 4, 641 (2010).

${ }^{4}$ L. Young, E. P. Kanter, B. Krässig, Y. Li, A. M. March, S. T. Pratt, R. Santra, S. H. Southworth, N. Rohringer, L. F. DiMauro, G. Doumy, C. A. Roedig, N. Berrah, L. Fang, M. Hoener, P. H. Bucksbaum, J. P. Cryan, S. Ghimire, J. M. Glownia, D. A. Reis, J. D. Bozek, C. Bostedt, and M. Messerschmidt, Nature (London) 466, 56 (2010).
${ }^{5}$ J. P. Cryan, J. M. Glownia, J. Andreasson, A. Belkacem, N. Berrah, C. I. Blaga, C. Bostedt, J. Bozek, C. Buth, L. F. DiMauro, L. Fang, O. Gessner, M. Guehr, J. Hajdu, M. P. Hertlein, M. Hoener, O. Kornilov, J. P. Marangos, A. M. March, B. K. McFarland, H. Merdji, V. S. Petrovic, C. Raman, D. Ray, D. Reis, F. Tarantelli, M. Trigo, J. L. White, W. White, L. Young, P. H. Bucksbaum, and R. N. Coffee, Phys. Rev. Lett. 105, 083004 (2010)

${ }^{6}$ L. Fang, M. Hoener, O. Gessner, F. Tarantelli, S. T. Pratt, O. Kornilov, C. Buth, M. Gühr, E. P. Kanter, C. Bostedt, J. D. Bozek, P. H. Bucksbaum, M. Chen, R. Coffee, J. Cryan, M. Glownia, E. Kukk, S. R. Leone, and N. Berrah, Phys. Rev. Lett. 105, 083005 (2010).

${ }^{7}$ L. Hedin and G. Johansson, J. Phys. B 2, 1336 (1969).

${ }^{8}$ D. A. Shirley, Chem. Phys. Lett. 17312 (1972); D. A. Shirley, Phys. Rev. A 7, 1520 (1973).

${ }^{9}$ F. P. Larkins, J. Phys. B 7, 47 (1976).

${ }^{10}$ C. D. Wagner, Anal. Chem. 44, 967 (1972); C. D. Wagner, Anal. Chem. 47, 1201 (1975).

${ }^{11}$ G. Moretti, J. Electron Spectrosc. Relat. Phenom. 95, 95 (1998).

${ }^{12}$ H. Siegbahn and O. Goscinski, Phys. Scr. 13, 225 (1976).

${ }^{13}$ T. D. Thomas, J. Electron Spectrosc. Relat. Phenom. 20, 117 (1980).

${ }^{14}$ J. H. D. Eland, M. Tashiro, P. Linusson, M. Ehara, K. Ueda, and R. Feifel, Phys. Rev. Lett. 105, 213005 (2010).

${ }^{15}$ P. Lablanquie, F. Penent, J. Palaudoux, L. Andric, P. Selles, S. Carniato, K. Bučar, M. Žitnik, M. Huttula, J. H. D. Eland, E. Shigemasa, K. Soejima, Y. Hikosaka, I. H. Suzuki, M. Nakano, and K. Ito, Phys. Rev. Lett. 106, 063003 (2011)

${ }^{16}$ P. Linusson, O. Takahashi, K. Ueda, J. H. D. Eland, and R. Feifel, Phys. Rev. A 83, 022506 (2011).

${ }^{17}$ L. S. Cederbaum, F. Tarantelli, A. Sgamellotti, and J. Schirmer, J. Chem. Phys. 85, 6513 (1986).

${ }^{18}$ L. S. Cederbaum, F. Tarantelli, A. Sgamellotti, and J. Schirmer, J. Chem. Phys. 86, 2168 (1987).

${ }^{19}$ L. S. Cederbaum, Phys. Rev. A 35, 622 (1987).

${ }^{20}$ E. M.-L. Ohrendorf, L. S. Cederbaum, and F. Tarantelli, Phys. Rev. A 44, 205 (1991).

${ }^{21}$ H. Ågren and H. J. Aa. Jensen, Chem. Phys. 172, 45 (1993).

${ }^{22}$ C. Reynaud, M.-A. Gaveau, K. Bisson, Ph. Millié, I. Nenner, S. Bodeur, P. Archirel, and B. Lévy, J. Phys. B 29, 5403 (1996).

${ }^{23}$ M. Tashiro, M. Ehara, H. Fukuzawa, K. Ueda, Ch. Buth, N. V. Kryzhevoi, and L. S. Cederbaum, J. Chem. Phys. 132, 184302 (2010).

${ }^{24}$ M. Tashiro, M. Ehara, and K. Ueda, Chem. Phys. Lett. 496, 217 (2010).

${ }^{25}$ T. H. Dunning, Jr., J. Chem. Phys. 90, 1007 (1989); R. A. Kendall, T. H. Dunning, Jr., and R. J. Harrison, J. Chem. Phys. 96, 6796 (1992).

${ }^{26}$ M. J. Frisch, G. W. Trucks, H. B. Schlegel et al., GAUSSIAN 03, Revision D.02, Gaussian, Inc., Wallingford, CT, 2004.

${ }^{27}$ J. Schirmer, L. S. Cederbaum, and O. Walter, Phys. Rev. A 28, 1237 (1983).

${ }^{28}$ J. Schirmer and A. Barth, Z. Phys. A 317267 (1984).

${ }^{29}$ W. J. Hehre, R. Ditchfield, and J. A. Pople, J. Chem. Phys. 56, 2257 (1972); P. C. Hariharan and J. A. Pople, Theoret. Chimica Acta 28, 213 (1973).

${ }^{30}$ P. S. Bagus, Phys. Rev. 139, A619 (1965).

${ }^{31}$ S. Faas, J. G. Snijders, J. H. van Lenthe, E. van Lenthe, and E. J. Baerends, Chem. Phys. Lett. 246, 632 (1995).

${ }^{32}$ J. H. van Lenthe, S. Faas, and J. G. Snijders, Chem. Phys. Lett. 328, 107 (2000).

${ }^{33}$ The original $\mathrm{ADC}(4)$ code for core ionization was written by J. Angonoa and J. Schirmer; contributions due to F. Tarantelli and N. V. Dobrodey.

${ }^{34}$ F. Tarantelli, Chem. Phys. 326, 11 (2006).

${ }^{35}$ M. F. Guest, I. J. Bush, H. J. J. van Dam, P. Sherwood, J. M. H. Thomas, J. H. van Lenthe, R. W. A. Havenith, and J. Kendrick, Mol. Phys. 103, 719 (2005).

${ }^{36}$ T. Helgaker, W. Klopper, H. Koch, and J. Noga, J. Chem. Phys. 106, 9639 (1997).

${ }^{37}$ V. Myrseth, K. J. Børve, K. Wiesner, M. Bässler, S. Svensson, and L. J. Sæthre, Phys. Chem. Chem. Phys. 4, 5937 (2002).

${ }^{38}$ See supplementary material at http://dx.doi.org/10.1063/1.3624393 for the computed inner-shell single and double ionization potentials of the phenol and aniline molecules.

${ }^{39}$ T. X. Carroll, T. D. Thomas, H. Bergersen, K. J. Børve, and L. J. Sæthre, J. Org. Chem. 71, 1961 (2006); V. Myrseth, L. J. Sæthre, K. J. Børve, and T. D. Thomas, J. Org. Chem. 72, 5715 (2007); T. X. Carroll, T. D. Thomas, L. J. Sæthre, and K. J. Børve, J. Phys. Chem. A 113, 3481 (2009).

${ }^{40}$ K. A. Peterson and T. H. Dunning, Jr., J. Chem. Phys. 117, 10548 (2002). 